\title{
Availability Design of Optical Transport Networks
}

\author{
Massimo Tornatore, Student Member, IEEE, Guido Maier, Member, IEEE, and Achille Pattavina, Senior Member, IEEE
}

\begin{abstract}
A design technique for reliable optical transport networks is presented. The network is first dimensioned in order to carry a given set of static protected optical connections, each one routed maximizing its availability. The network can be further optimized by minimizing the number of fibers to be installed, while keeping a control on connection availability, which can remain the same or decrease by a prefixed margin factor. Design and optimization algorithms are provided for networks adopting dedicated and shared path-protection. The optimization approach is heuristic. Results obtained by applying the proposed technique to two casestudy networks are shown and discussed. These two case-study experiments are carried out exploiting a realistic model to evaluate terrestrial and submarine optical link availability.
\end{abstract}

Index Terms-Deterministic network calculus, mathematical programming/optimization, system design.

\section{INTRODUCTION}

$\mathbf{T}$ HE ADVENT of broadband services led to an extremely rapid growth of traffic. Optical transport networks (OTNs) are the most efficient solution to quench the future bandwidth demand. Network reliability and protection are among the most important issues concerning the transport of high-speed connections: interruption of an optical connection even for a short period (e.g., a second) could cause the loss of a huge quantity of data (e.g., 5 GByte for a 40 Gbit/s-modulated wavelength channel).

The guarantees of a prefixed maximum outage time and a maximum connection-failure probability [1] for a connection become fundamental components of the service level agreement between an operator and its customers. Being an OTN system composed of reparable subsystems, quality is usually measured in terms of connection availability (a measure of outage time), rather than reliability (a measure of disconnection probability), though this second parameter could have its relevance [2]. Many authors have proposed various ad hoc reliability parameters to be employed to networking problems [1], [3]-[5]. In this paper, we will refer to availability defined according to classical reliability theory [6], [7]. The method proposed could however apply without major changes also to cases in which reliability is the major concern.

Even if optical transmission systems and OTN switching nodes have reached high or at least acceptable technological quality, trying to satisfy customers' requirements by relying

Manuscript received December 23, 2003; revised December 23, 2004. This work was supported in part by MIUR, Italy, under FIRB Project ADONIS and in part by EU IST Network of Excellence e-Photon/One.

M. Tornatore and A. Pattavina are with the Department of Electronics and Information, Politecnico di Milano, Milan 20133, Italy (e-mail: tornator@elet.polimi.it; pattavina@elet.polimi.it).

G. Maier is with CoreCom, Milan 20133, Italy (e-mail: maier@corecom.it).

Digital Object Identifier 10.1109/JSAC.2005.851774 only upon this aspect would be too expensive for the operator. Redundancy is the technique that is commonly exploited to meet reliability and availability requirements. In the context of OTN, redundancy is implemented by adopting optical protection techniques. Usually, these are based on the concept that each connection has a backup path to recover it in case of failure. Obviously, protection requires available network resources and, thus, an extra cost for the operator [8].

In this pasper, we will consider planning of an OTN starting from a known set of optical connection requests and from a greenfield physical installation. The sets of nodes and of wavelength-division-multiplexing (WDM) links connecting them are given, together with the physical lengths of the links. Each connection is end-to-end protected, i.e., it is implemented by setting up a pair of lightpaths from the source to the destination node, one of which is used in working conditions and the other is for protection. Network planning consists in allocating resources (in terms of WDM channels) to both working and protection lightpaths. Two optimization objectives are pursued: 1) maximization of the availability level guaranteed to the users and 2) minimization of the total number of fibers that must be installed to deploy the network (assuming that every fiber carries the same prefixed number of WDM channels). We will show different strategies to achieve these objectives: initially, no availability target is specified and all the connections are individually routed so to maximize the availability of each one, with no constraints on physical network resources; then the total number of fibers is minimized, by assuming the availability values obtained by the first strategy as constraints; finally, physical-resource minimization is repeated in the same way as before, but relaxing the availability constraints by a prefixed ratio.

We developed a design procedure, based on heuristic optimization, that can be applied to both the two possible implementations of end-to-end OTN protection, which are: dedicated path protection (DPP) and shared path protection (SPP). Most of the recent works published in the area of OTN availability design covered only DPP [4]-[7], [9]. Some studies have been recently published to analyze availability in SPP scenario: authors in [10] and [11] investigate how setting a limit on the number of connections that share the same pool of backup resources can improve the average connection availability in the network, without causing a significant paying off in capacity requirements; [12] propose to use connection availability as a metric to provide differentiated protection service, recurring to different protection strategies, under a static traffic assignment; novel availability-aware strategies for dynamic traffic have been proposed in [13] and [14], where authors exploit availabilitybased metric to take on-the-fly routing decision. In this paper, we will focus on network design based on static traffic matrix. We believe that our systematic approach for a joint availability 
analysis and optimization with shared protection resources is an innovative contribution of the work we are presenting.

This paper has been organized as follows. In Section II, some basic elements of availability and reliability theory, useful to the subsequent analysis, are briefly reviewed. In Section III, we illustrate the algorithms used by our optimization procedure, for dedicated and shared protection, discussing at the end a possible extension of the paper to include shared risk link groups. Section IV describes the reference availability model of WDM channel we have adopted to obtain the availability data for the case-study networks. In Section V, two examples of network planning are shown and the results obtained are compared and discussed.

\section{Availability ANALYSIS}

Reliability theory [2], [15] gives the instruments to calculate the reliability parameters of a complex system. The starting point of such evaluation is availability characterization of the system functional blocks. Generally speaking, any system functional block can be associated to an instantaneous rate of failure $z(t)$. This function is evaluated by defining a suitable reliability model of the functional block. Parameters of the model are commonly specified by technology vendors or may be identified by experimental testing before system installation and then they may be updated by field measurements during the system operating lifetime.

In our case, the system to be characterized is the optical connection, which comprises as functional blocks the WDM channels and the nodes on which it is routed. In this paper, we have, however, considered ideal OTN nodes, i.e., perfectly reliable and immune from any kinds of failure (assumption not far from reality, according to [4]). Thus, only WDM channels have to be taken into account as functional blocks. A WDM channel is part of an OTN link, composed of the fiber cable installed between two adjacent nodes and equipped by a set of line devices (e.g., optical amplifiers). The details of the reliability model adopted to describe a WDM channel will be given in Section IV; at this point, we shall only say that the model is based on the usual approximation of considering a constant rate of failure $z(t)=\lambda$, corresponding to a negative exponential reliability function $R(t)=e^{-\lambda t}$. According to such approximation, the mean time to failure (MTTF) of a WDM channel is simply given by $\mathrm{MTTF}=1 / \lambda$. Moreover, this kind of model implies that the WDM channels of a given optical connection are mutually failure-independent and that failures randomly occur in time, independently from the components age. Another parameter has to be introduced, which is the mean time to repair (MTTR) of a WDM channel, again assumed to be constant in time. We introduce also the mean time between failures (MTBF) parameter, which is given by MTBF $=\mathrm{MTTF}+\mathrm{MTTR}$.

Since we are interested in availability and we consider WDM links as reparable systems, system availability can be measured by the so called average steady-state availability $A$ (indicated simply as availability in the rest of the paper for short). This parameter corresponds to the probability that the system is able

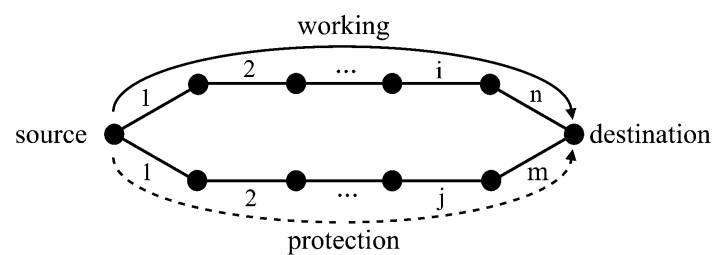

Fig. 1. Availability scheme of a path-protected optical connection.

to perform its function at the time it is requested to do so, or, equivalently, to its average outage time. It can be shown [2] that for each functional block of the system the following relation holds:

$$
A=\frac{\mathrm{MTTF}}{\mathrm{MTTF}+\mathrm{MTTR}}=\frac{\mathrm{MTTF}}{\mathrm{MTBF}} .
$$

We shall also introduce, for sake of presentation, the complement of $A$, which is the average steady-state unavailability $U=$ $1-A$ (unavailability for short)

$$
U=\frac{\text { MTTR }}{\mathrm{MTTF}+\mathrm{MTTR}}=\frac{\mathrm{MTTR}}{\mathrm{MTBF}} .
$$

Fig. 1 shows the scheme of an optical connection protected by DPP. The working and protection lightpaths are represented by displaying the set of WDM channels on which they are routed.

The basic condition of end-to-end protection is working and protection lightpaths (the w/p pair) being routed along failureindependent network elements. Given that nodes have been assumed ideal, the failure event we are considering in this paper is link failure. When it occurs, all the WDM channels belonging to the failed link simultaneously become unavailable. Therefore, the above condition of independency translates in our case to the simple constraint that the working and the protection lightpaths must not be routed on WDM channels belonging to the same link, or, synthetically, it reduces to a link-disjoint routing condition for the w/p pair. ${ }^{1}$ Given the absence of failure correlation implied by link-disjointness, the availability of the working and the protection lightpaths can be individually computed. Each of the two lightpaths is the series of its WDM channels. According to the approximate model adopted, the availability of the working lightpath is given by the following equation:

$$
A_{w}=\prod_{i=1}^{n} A_{i}
$$

where $i$ indicates the $i$ th of the $n$ WDM channels composing the working lightpath.

For realistic systems, $A_{i} \approx 1$. The following useful approximate equation will then be employed to evaluate lightpath unavailability [5]

$$
U_{w}=1-A_{w}=1-\prod_{i=1}^{n}\left(1-U_{i}\right) \approx \sum_{i=1}^{n} U_{i} .
$$

Similar equations apply to the protection lightpath in order to evaluate $A_{p}$ (or $U_{p}$ ).

\footnotetext{
${ }^{1}$ In order to analyze the effects of this hypothesis, we will discuss possible extensions of our methodology to shared risk link groups in Section III-D.
} 
Since we have identified the single optical connection as the system to be characterized in terms of availability, we should clarify in which conditions it is available. Being it is implemented by end-to-end protection, a connection is "on" when either the working, the protection or both the lightpaths are available. In DPP, the state of availability of the two lightpaths does not depend upon the state of any other lightpath: each DPP connection can be treated separately from all the other connections in the network, applying the simple methods proposed by Lee [16] for blocking probability evaluation due to traffic congestion, a field that can be considered dual to reliability, in the sense that mathematical formulations are in general the same if we substitute congestion to reliability (or availability) parameters. Hence, the total availability of the DPP connection $c$ is obtained by considering the parallel of working and protection lightpaths [4] according to the following simple equation:

$$
U_{c}=U_{w} \cdot U_{p}
$$

Analysis of connection availability gets more complicated in case of SPP. When a connection shares protection resources with others, the possibility of exploiting its spare lightpath in case of failure on the working lightpath depends on the state of the other sharing connections. Lee's approach could be still applied to compute approximate values, but then results would be too far from reality to be useful [17]. In order to assess availability of the connection with sufficient accuracy, combinations of multiple failure events should be taken into account: the list of such combinations becomes rapidly lengthy as the number of shared WDM channels and the number of sharing connections increase. We have however proposed a simplified analysis that leads to a closed-form equation (details are reported in [18]). The proposed approximation method consists in neglecting some multiple-failure combinations that are favorable to the recovery of the connection for which availability is being estimated.

Let us consider a generic connection $c$. The sharing group of $c$ is the set $\mathbf{C}$ of all the connections $i \neq c$ that have their protection lightpaths sharing one or more WDM channels with the protection lightpath of $c$. This set, composed by $|\mathbf{C}|=m$ elements, is such that all the working lightpaths of its members are failure-disjoint with the working lightpath of $c . c$ is "on" when its working lightpath is "on"; $c$ is "on" also when its working lightpath is "off," the unshared links of its protection lightpath are "on" and no connection of $\mathbf{C}$ is using the shared part of the protection lightpath of $c$. This latter condition is certainly true (sufficient condition) when each $i \in \mathbf{C}$ has its working lightpath "on."

Our simplified approach considers only the events listed above: being based on sufficient conditions it returns a conservative approximate availability value of connection $c$, given by

$$
A_{c} \approx A_{w_{c}}+\left(1-A_{w_{c}}\right) A_{p_{c}} \prod_{i=1}^{m} A_{w_{i}}
$$

$A_{w_{i}}$ and $A_{p_{i}}$ are the availability of the working and the protection lightpath, respectively, of a generic connection $i$ of the sharing group $\mathbf{C}$ of $c ; A_{w_{c}}$ and $A_{p_{c}}$ are the availability of working and protection lightpaths of $c$ itself.
In order to asses the availability of $c$ without approximation, we should comprise other possible failure patterns, all regarding the case in which $w_{c}$ is "off." In order to go through more details, let us assume that a priority-criterion is adopted in the sharing group C. $\mathbf{C}$ is divided into the two disjoint subsets $\mathbf{C}^{+}$and $\mathbf{C}^{-}$: if the working lightpath of any $i \in \mathbf{C}^{+}$fails along with the working lightpath of $c$, then the protection resources shared among $c$ and $i$ are exploited to recover $i$, while $c$ is lost. The opposite happens when $w_{j}$ fails with $j \in \mathbf{C}^{-}$. In the favorable event-combinations for $c$ survivability, all the cases in which $w_{c}$ is "off" together with one or more working lightpaths of the connections of $\mathbf{C}^{-}$should be accounted. Of all these combinations, those with a single $w_{j}$ in failure with $w_{c}$ would add the maximum contribution to $c$ availability (two failures $\rightarrow$ second-order contribution). In [18], exploiting Monte Carlo simulation, we have verified that, for realistic availability values of WDM links, by neglecting such contributions our approximate analysis overestimates the average unavailability of a connection by less than $1 \%$, if in the real network a fair contention-resolution method is adopted.

Let us consider now the case in which $w_{c}$ is "off" together with $w_{i}$, with $i \in \mathbf{C}^{+}$. There may be one or more failures hitting the links of $p_{i}$ which are not shared with $p_{c}$, leaving the shared parts intact: the consequence is that, though the shared parts of $p_{c}$ should have been used to recover $i$, they can be reverted to the recovery of $c$, as $i$ is out-of-play. The same easily extends to the cases in which more than one connection of $\mathbf{C}^{+}$is in the same conditions of $i$. The most relevant to $c$ availability of these event combinations are those involving $c$ and only one connection $i \in \mathbf{C}^{+}$: they are three-failure events $\rightarrow$ third-order contribution. Reference [18] shows that neglecting them leads to differences between real and approximate connection availability of less than $0.01 \%$ for realistic values of WDM links availability.

Our approximation, in short, consists in neglecting all failure event combinations in which $w_{c}$ fails together with one or more $w_{i}$ with $i \in \mathbf{C}$ : this implies to assume that all the connections involved in multiple working-lightpath failures are lost, leaving the protection resources unused. Our approximate model is slightly pessimistic, with the advantage however that backup-resource contention does not have to be included into the model and the list of events to be considered is limited to a tractable size.

Equation (4) clearly shows that the availability of an SPP connection depends on the availabilities of its own w/p pair and of just the working lightpaths of all the other connections of the sharing group. Sharing-group cardinality is a main parameter of the equation, while the number of protection WDM channels that are actually shared has no relevance. Note that (4) reduces to (3) in the case $m=0$ (no sharing, thus, DPP) and obviously it returns the exact result without approximations.

Let us consider the example shown in Fig. 2, in which a portion of a network is shown hosting the sharing group $\mathbf{C}$ of $c$. It should be noted that all the working lightpaths of the group are link-disjoint, as a consequence of the basic condition of shared protection, which is that all the connections of a sharing group must always be recoverable from a single link-failure event. In the example, the availability of connection $c$ is $A_{c} \approx$ $A_{w_{c}}+\left(1-A_{w_{c}}\right) A_{p_{c}} \cdot\left(A_{w_{1}} \cdot A_{w_{2}} \cdots A_{w_{m}}\right)$. 


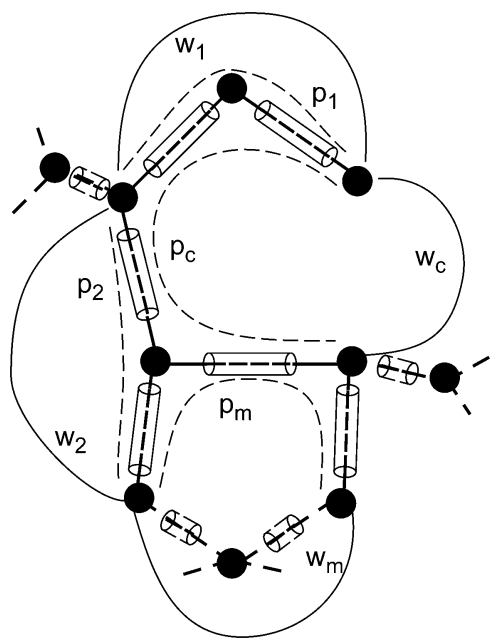

Fig. 2. Sharing group $\mathbf{C}$ of the optical connection $c$.

To conclude this section, let us add a final general remark. In this paper, we have considered only techniques able to recover single-link failures. More complicated protection techniques would allow recovery of simultaneous and independent multiple-link failures affecting both the working and the protection lightpath of a w/p pair. Multiple-failure recovery [19] greatly improves (by orders of magnitude) the connection availability [18], but on the other hand it requires a much higher network deployment cost. We will show later in this paper that, if we consider only internal failure causes, single-failure recovery is more than sufficient to meet the five-nines availability target usually demanded by customers at affordable deployment costs.

\section{DESIGN AND OptimizATION PROCEDURE}

Let us now describe our availability-design method. As we have stated in Section I, network optimization is carried out in two phases according to two different cost functions: connection availability and fiber number. The first phase is called maximum-connection-availability design (MCAD). It solves the problem of allocating resources to each of the demanded connections in an unconstrained-capacity network. The second phase is named availability-constrained physical-resources optimization (ACPRO).

\section{A. MCAD—Dedicated Path Protection}

MCAD is solved with a heuristic technique by allocating resources for all the connection requests in sequence, starting from an empty network with oversized number of fibers on all the links, so that the amount of physical resources is never a constraint. Since the number of wavelengths per fiber is an input parameter, identical for all the fibers of the network, a safe oversize method is to initially set the number of fibers per link equal to $R_{c} / W$, where $R_{c}$ is the total number of connection requests and $W$ is the number of wavelengths per fiber.

Allocating resources to a protected connection is equivalent to solving routing and fiber and wavelength assignment (RFWA) for its working and protection lightpath (under the link disjoint constraint). To solve this problem, we have exploited a tool previously developed for WDM network design, the details of which have been published elsewhere [20], [21], and will be omitted here for brevity. Availability-oriented design capability has been introduced as a new feature of the tool. The principle of the tool is to transform the RFWA problem into a minimum-weight path-search problem on a multifiber layered graph (MLG). This representation of the network is obtained by including one arc per WDM channel in the graph; arcs are grouped so as to form a stack of layers, one per wavelength and $W$ per fiber; layers are connected by vertical arcs at the nodes, to represent wavelength conversion or switching possibilities; an arc allocated to a lightpath is marked as busy and cannot be reallocated. The arcs of the graph are assigned suitable weights, so that the minimum-weight path-searching algorithm can reproduce the selection operated by some RFWA criteria, such as shortest-path routing, least-loaded routing, first-fit wavelength assignment, and so on. In case of end-to-end protection, the path search is substituted by an algorithm which finds resources to the w/p pair lightpaths under the link-disjoint constraint.

The above method can be easily extended to availability design simply by assigning each arc of the MLG a weight equal to the unavailability value of the corresponding WDM channel. Section IV explains how these values are obtained. Some changes have however to be made upon the algorithms operating on the MLG, that are different for DPP and SPP.

From (3), we know that the unavailability of a DPP connection is $U_{c}=U_{w} \cdot U_{p}$. The most available RFWA in this case is the cycle in the idle portion of MLG that minimizes $U_{c}$. Such a cycle could be found by nonliner programming, with a high computational complexity. We propose instead the following heuristic method [9]. Two known algorithms can be applied to find a cycle on a weighted graph: the "one-step" (or Bhandari [22]), finds the link-disjoint pair of paths having the minimum total weight $\left(\min \left\{U_{w}+U_{p}\right\}\right)$; the "two-step" (or repeated Dijkstra), assigns as working the least-unavailability path $\left(\min \left\{U_{w}\right\}\right)$ and as protection the second link-disjoint least-unavailability path $\left(\min \left\{U_{p} \mid U_{p} \cap U_{w}=\emptyset\right\}\right)$. None of the two algorithms actually minimizes $U_{c}$, but the suboptimal solutions they find are expected to be very close to the absolute optimum. Our method applies both the algorithms for each connection and keeps the solution found which gives the lowest unavailability. It can be proved that when the two solutions are identical, they also coincide with the actual optimum [23]: since the two-step approach minimizes the unavailability $U_{w}$ and the one-step approach minimizes the sum $U_{w}+U_{p}$, we prove that, when they coincide, also the product $U_{c}=U_{w} \cdot U_{p}$ is minimized. The proof of this statement shows that the hyperbola associated to the product $U_{w} \cdot U_{p}=K$ in the plane $U_{w}-U_{p}$ lies at the minimal value of $K$ in correspondence of the intersection between the two lines representing, respectively, $\min \left\{U_{w}\right\}$ and $\min \left\{U_{w}+U_{p}\right\}$.

We conjecture that this heuristic approach gives results sensibly far from the optimum only in extremely connected networks.

Once all the connection requests have been satisfied, empty fibers are removed from the network.

\section{B. MCAD-Shared Path Protection}

The heuristic method described for DPP cannot be applied in the case of SPP because the "one-step" algorithm does not work. Bhandari algorithm jointly builds the two lightpaths of the w/p 
pair, but when protection resources are shared, the knowledge of the working lightpath is needed to build the protection in order to check link disjointness of the working lightpaths of the same sharing group.

We have, therefore, developed another heuristic algorithm which is divided in the following steps.

Step 1) Start with the network idle and oversized.

Step 2) Take the first not-yet satisfied connection request $c$ of the sorted list of connection requests.

Step 3) Find RFWA of the working lightpath $w_{c}$ applying Dijkstra on the MLG, using WDM channel unavailability as weights.

Step 4) Build the list $\mathbf{Y}(c)$ of already allocated connections having their working lightpaths sharing at least a link with $w_{c}$.

Step 5) Disable links crossed by $w_{c}$.

Step 6) Find RWFA of the protection lightpath $p_{c}$ applying Dijkstra on the remaining MLG, using WDM-channel unavailability as weights. An arc of the MLG can be allocated to $p_{c}$ only if it is not already allocated to a protection lightpath of a connection belonging to $\mathbf{Y}(c)$.

Step 7) Set to zero all the weights of the arcs belonging to $p_{c}$. This operation is required to induce future protection lightpaths to share WDM channels with $p_{c}$.

Step 8) Remove empty fibers and end if all the connection requests have been satisfied; else go to Step 2).

Note that the use of (4) is not necessary in MCAD.

\section{ACPRO-Dedicated and Shared Path Protection}

MCAD does not care about network resources. In particular, at the end of MCAD many partially empty fibers will be present in the network, since MCAD is "greedy" under the fiber occupation point of view. After the MCAD phase, ACPRO gives the possibility of decreasing the number of installed fibers while keeping connection availability under control. ACPRO is transparent to the kind of protection adopted and, thus, works the same with DPP and SPP.

A margin $M$ is fixed as an input to ACPRO: the optimization procedures guarantees that the final unavailability of each connection is less then $M$ times the unavailability reached for that connection after MCAD. The second input to ACPRO is the network with all the connections allocated as resulting from MCAD.

The optimization procedure is displayed in the flowchart of Fig. 3. The following symbols are used in the chart:

- $K$ is a counter running from 0 to $W-1$, where $W$ is the number of wavelengths per fiber (a known global network parameter);

- a $K$-fiber is a fiber having $K$ WDM channels allocated to working or protection lightpaths;

- $f$ is the index denoting the currently processed fiber;

- $\mathbf{X}(f)$ is the set of connections, each one such that either its working or its protection lightpath crosses fiber $f$;

- $\boldsymbol{\Omega}[\mathbf{X}(f)]$ denotes a particular RFWA solution for the w/p pairs of all the connections of $\mathbf{X}(f)$;

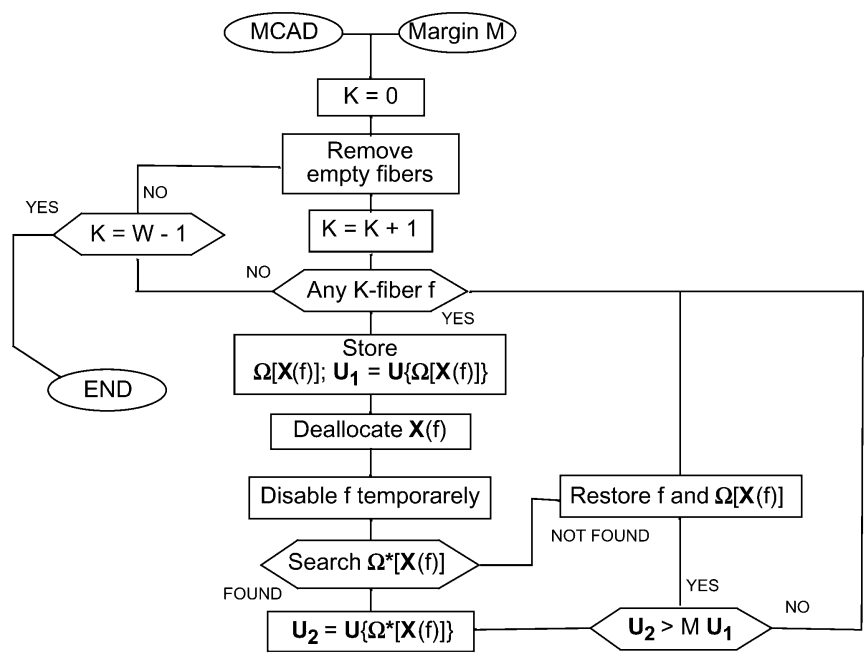

Fig. 3. Flow chart of the ACPRO procedure.

- $\mathbf{U}\{\boldsymbol{\Omega}[\mathbf{X}(f)]\}$ is the set of unavailability values of all the connections of $\mathbf{X}(f)$ routed according to $\boldsymbol{\Omega}[\mathbf{X}(f)]$. It is obtained by solving (4) for all the connections of the set.

ACPRO starts by removing all the already empty fibers (i.e., $K$-fibers with $K=0$ ). $K$ is incremented and the first $K$-fiber with the new value $K$ is detected. Let us indicate such a fiber by the index $f$. A reallocation routine is then performed on fiber $f$ : the current RFWA solution $\Omega[\mathbf{X}(f)]$ of the connections of the set $\mathbf{X}(f)$ is stored, together with their unavailability values $\mathbf{U}_{\mathbf{1}}=\mathbf{U}\{\mathbf{\Omega}[\mathbf{X}(f)]\} ; \mathbf{X}(f)$ are deallocated (the resources their $\mathrm{w} / \mathrm{p}$ pairs were occupying are freed) and they are rerouted after having disabled $f$. If rerouting is not possible for all the deallocated w/p pairs due to lack of free resources, then $f$ is put in place again and the old network state $\boldsymbol{\Omega}[\mathbf{X}(f)]$ is restored. If an alternative RFWA $\Omega^{*}[\mathbf{X}(f)]$ exists (all the connections of $\mathbf{X}(f)$ can be rerouted), the corresponding set of unavailability values $\mathbf{U}_{\mathbf{2}}=\mathbf{U}\left\{\boldsymbol{\Omega}^{*}[\mathbf{X}(f)]\right\}$ is computed and compared with $M \cdot \mathbf{U}_{\mathbf{1}}$ : the new solution is accepted and $f$ permanently removed only if the unavailability of any connection has not increased by more than a factor $M$; else, $f$ and the old $\boldsymbol{\Omega}[\mathbf{X}(f)]$ are restored. Once the reallocation routine is concluded on $f$, the next $K$-fiber is detected and it undergoes the deallocation routine. The process is repeated until all the $K$-fibers have been probed. Then, $K$ is incremented and the entire cycle repeated until $K=W-1$. At the end, all the partially used fibers have been probed at least once.

\section{Extension to Shared Risk Link Group (SRLG)}

By definition, a shared risk group (SRG) [24] is a set of network elements that are simultaneously struck by the same failure event. Since nodes have been assumed ideal, the approach presented in this paper could be extended to consider the presence of shared risk link group (SRLG) [25], [26] in the network. Commonly, SRLGs occur only in very particular geographical conditions, such as when the only affordable opportunity to connect an island is through a single bridge which must thus be shared by all the cables: they may, however, be of interest in network planning.

When a failure occurs on a SRLG, all the WDM channels belonging it simultaneously become unavailable. Therefore, 
the condition of independency between the working and the protection lightpaths of a connection used in Section II must be extended from the simple link-disjoint constraint to the SRLG-disjoint routing condition. Let us discuss what is the impact of this generalization on the design and optimization procedures explained above.

Routing a SRLG-disjoint w/p pair with a "one-step" method is not possible in a general case, even in the simple DPP case [22]. The presence of SRLG's sets correlations between physically disjoint links: the knowledge of the routing of the working lightpath is needed to build the protection in order to check SRLG disjointness, in a similar way as it happens in the link-disjoint SPP case. In [22], Bhandary provides modifications of the one-step method that can be used with some SRLG configurations, e.g, the fork and the express link configuration. However, if a "two-step" approach is always adopted, then the extension becomes easy.

In the SPP scenario, MCAD can be applied to the SRLG case with no major modifications, since only the "two-step" approach is already exploited also for the link-disjoint constraint. Steps 4) and 5) reported in Section III-B have to be modified as follows: 1) build the list $\mathbf{Y}(c)$ of already allocated connections having their working lightpaths sharing at least a SRLG with $w_{c}$ and 2) disable all the links of the SRLGs crossed by $w_{c}$.

In Section III-A, we have explained that our MCAD procedure relies both on a "one-step" and a "two-step" path-finding algorithm. The extension of to planning in presence of SRLG's is simply achieved by giving up the "one-step" part and performing just the repeated Dijkstra. The possibility of verifying if a connection is actually routed along the least-unavailable cycle is no longer available. However, as already mentioned, our experience in the link-disjoint case with realistic network topology is that situations in which the "two-step" approach returns results different from the "one-step" are quite rare. Finally, the exploration of different alternative candidates, as shown in [27], can provide good results, alleviating the suboptimality of the "two-step" approach.

The ACPRO procedure is equivalently applicable to link-disjoin, as well as the SRLG-disjoint case, with the only care that rerouting shall be carried out according to the SRLG-disjoint constraint.

We are not going to deal any further with the SRLG case, limiting the rest of this paper to the link-disjoint case. In this section, we wanted to assess the potentiality of the proposed planning method to be extended and generalized. Its actual application to SRLG planning and its possible refinements for this purpose are currently under study.

\section{WDM CHANNEL AVAILABILITY MODEL}

To evaluate the WDM channel availability from component availability parameters, we employed the reference model depicted in Fig. 4. The figure shows a particular WDM channel belonging to a unidirectional fiber and the corresponding equipment involved in transmission.

The great majority of OTN switching nodes installed and operating today are actually electronic nodes: they perform cross-connecting of optical circuits after signals have been converted to an electrical format via suitable input-output

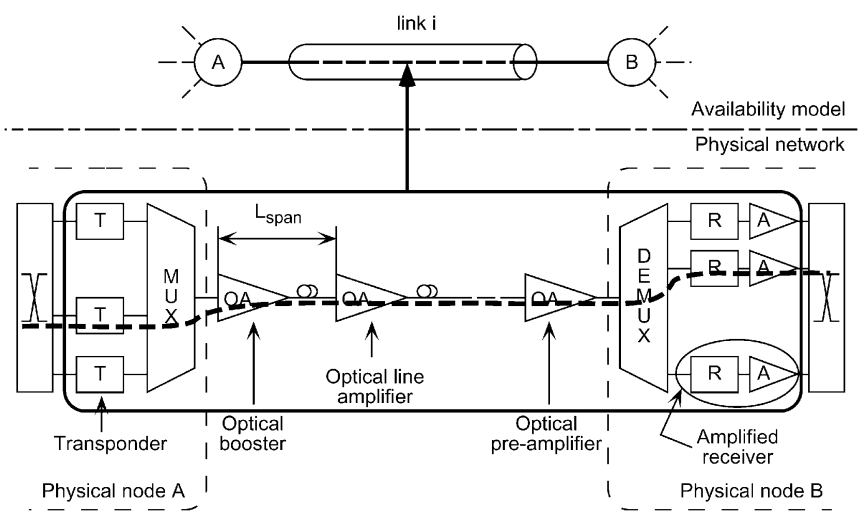

Fig. 4. Reference model of a WDM channel used to evaluate its availability.

interfacing cards. In this paper, we have considered all the nodes of the network to conform to this architecture. Therefore, each WDM channel is electronically terminated at both the nodes it connects. By the way, this implies that all the nodes are capable of performing wavelength conversion on all the transit lightpaths.

Our method can be equally applied to networks equipped by transparent optical cross connects, possibly with no or limited wavelength conversion capability. We have actually performed the same design experiments we are going to show next also on networks without wavelength conversion. Since such scenario is still beyond the experience of real network operators, it was impossible to find realistic availability data on transparent OXCs. We, thus, employed the same data for the opaque network we are going to show, simply including the wavelength continuity constraint in the planning process. The obtained results are very close to those reported below, indicating that wavelength conversion brings low advantages in static-traffic network planning.

Under the electric-node assumption, the WDM channel is modeled as series of components. Going downstream from the upstream termination the first component of the WDM channel is the transponder. We have assumed that the nodes are of the opaque type with uncolored optical outputs (all the signals at the same wavelength) or transmitting on a proprietary set of wavelengths: the transponder adapts the wavelength by converting it to a wavelength belonging to the set of the WDM multiplex suitable to be transmitted. The next components are the WDM multiplexer, the fiber, the chain of optical amplifiers with the segments of fiber connecting them, and the WDM demultiplexer: all these are shared by all the WDM channels of the fiber. The last component is a receiver with its amplification electronic circuitry.

By comparing operators' requirements and vendors' specifications (with the help of Pirelli Submarine Optical Systems) we have identified a set of availability values, applicable to the components of WDM channels belonging to a terrestrial or a submarine link. Data are reported in Table I. Only the line section of the link is differentiated between terrestrial and submarine, since submarine links are usually terminated onshore.

The MTTR depends upon environmental factors and also upon the operator's investments in the services of maintenance of network equipment. Reasonable ranges are: from 2 to $8 \mathrm{~h}$ for terrestrial link components, from 2 to 3 weeks for submarine 
link components (actual undersea reparations could take much longer, due to unexpected events such as sea storms). Note that line components of submarine links have much higher MTBF compare with the terrestrial counterparts, which partially compensates the higher MTTR. Also, the distance between two neighbor optical amplifiers (amplifier span $L_{\text {span }}$ ) for undersea cables is shorter than for a terrestrial link.

Given the availability of all the components of the series, it is trivial to evaluate the availability of the WDM channel $i$ by applying (2)

$$
U_{i}=U_{t x}+U_{r x}+\left[\operatorname{round}\left(\frac{L}{L_{\mathrm{span}}}\right)-1\right] \cdot U_{\mathrm{span}}
$$

where $U_{t x}=U_{\text {transp }}+U_{\text {mux }}+U_{\text {booster }}, U_{r x}=U_{\text {pre }}+$ $U_{\text {demux }}+U_{\text {amp.rcv }}, L$ is the length of the link (in kilometers), and $U_{\text {span }}$ is the unavailability of a span of cable (usually negligible) and an optical line amplifier.

All the components included in our model have been characterized in terms of their intrinsic availability, accounting for failures exclusively due to internal causes. Externally provoked failures (construction-work machinery impacts for terrestrial links, anchor impact or fish bytes for undersea cables, etc.) are not considered, though taking them into account would not change our model in any fundamental way. This choice is determined by the difficulty of building statistical models of external failure agents and by the fact that only intrinsic availability usually appears in system specifications. On the other hand, there is no clear agreement in literature about the effective statistical relevance of external failures: accidental link breakups are reported to be rare events by some authors (1.24 min/year [28]) or relatively frequent by some others (136 cuts in the U.S. during 1997 [8], or 1.5 cuts per 1000 miles/year, typically used in the U.S. long-haul networks). Since we you are focusing on the internal failure causes and the consequent relative availability values, actual values of unavailability could be worse.

Rigorously, the event of internal failure on a WDM channel characterized by our model does not coincide with the event of link failure. A failure of a transponder or an amplified receiver concerns a single WDM channel, as well as a failure of an optical amplifier or a mux/demux involves only one fiber of a link. However, in the context of our model, such failures can be interpreted as link failures, since there are no protection mechanisms able to locally reroute a failed WDM channel onto another WDM channel of the same link. From the connection point of view, a WDM channel failure activates exactly the same mechanisms as a link failure. Our dimensioning technique is conservative: it does not consider that survivor idle WDM channels of a partially failed link are available resources that could be used to carry traffic. However, we are dealing with preplanned protection systems that do not require the knowledge of failure location: the exploitation of survivor WDM channels is more appropriate for dynamic connection restoration.

\section{CAse-Study Analysis}

The design procedure explained in the previous sections has been applied to realistic case-study networks. Most of the design experiments concern a network connecting the major cities

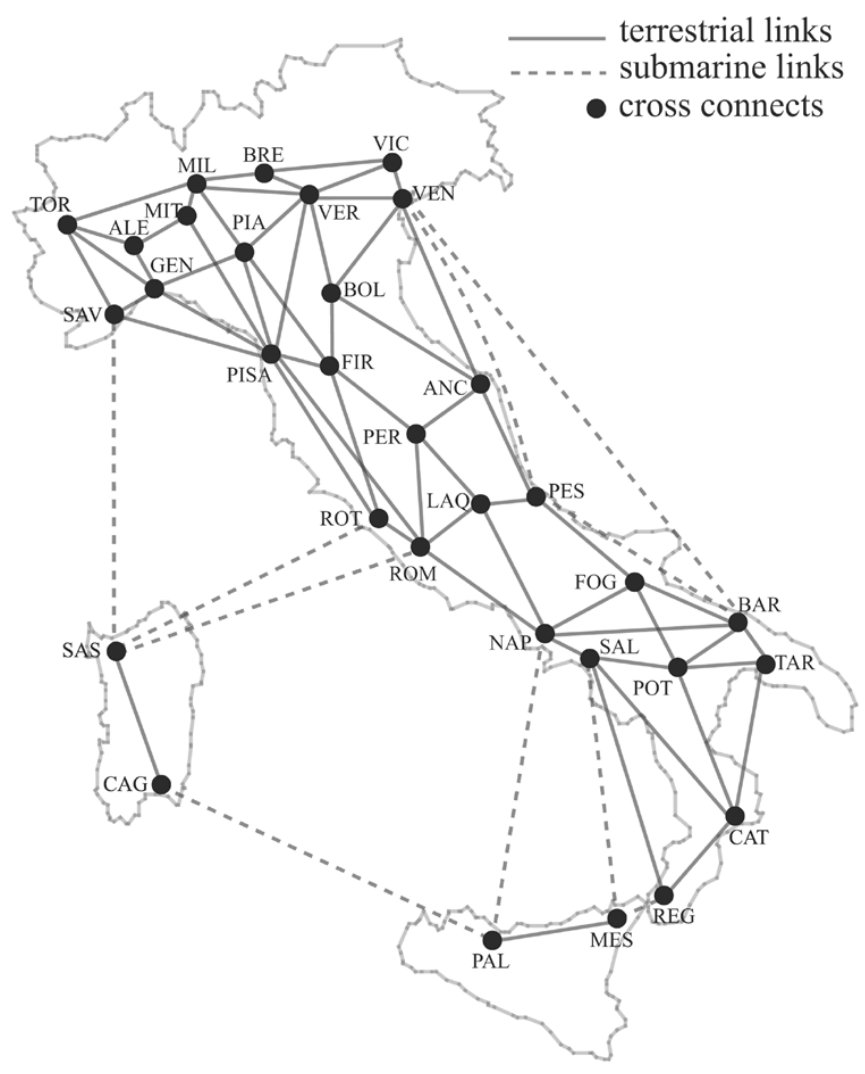

Fig. 5. Italian optical transport network (ITNet).

TABLE I

PARAMETERS OF DEVICES CONSIDERED IN THE WDM ChanNEL AVAILABILITY EVALUATION

\begin{tabular}{c|c|c|c}
\hline \multicolumn{5}{c}{ Transmitter side } \\
\hline Component & $M T B F$ khour & $M T T R$ hour & $U=1-A$ \\
\hline Transponder & 196 & 2 & $1.02 \cdot 10^{-5}$ \\
\hline Multiplexer & 606 & 2 & $3.30 \cdot 10^{-6}$ \\
\hline Booster & 211 & 2 & $9.48 \cdot 10^{-6}$ \\
\hline \multicolumn{4}{c}{ Receiver side } \\
\hline Component & $M T B F$ khour & $M T T R$ hour & $U=1-A$ \\
\hline Pre-amplifier & 370 & 2 & $5.40 \cdot 10^{-6}$ \\
\hline Demultiplexer & 279 & 2 & $7.17 \cdot 10^{-6}$ \\
\hline Amplif. receiver & 210 & 2 & $9.52 \cdot 10^{-6}$ \\
\hline \multicolumn{4}{c}{ Line (TERRESTRIAL LINK) } \\
$L_{\text {span }}=100 \mathrm{~km}$ \\
\hline Component & $M T B F$ khour & $M T T R$ hour & $U=1-A$ \\
\hline Amplifier & 211 & 2 & $9.48 \cdot 10^{-6}$ \\
\hline \multicolumn{4}{c}{ Line (SUBMARINE LINK) } \\
$L_{\text {span }}=57 \mathrm{~km}$ \\
\hline Component & $M T B F$ khour & $M T T R$ hour & $U=1-A$ \\
\hline Amplifier & $20 \cdot 10^{3}$ & 336 & $1.68 \cdot 10^{-5}$ \\
\hline
\end{tabular}

of Italy (Fig. 5). Though its physical topology does not exactly correspond to any commercial operator installation, it is a reasonable approximation of the real Italian optical backbone infrastructure. We have conventionally named it ITNet. It comprises 32 nodes and 72 links, 10 of which are submarine systems (dashed segments in Fig. 5).

The rather limited geographical extension of the network makes the terminal systems to have a greater influence on the WDM channel availability (evaluated by (5), using data of Table I) than the line systems. For the same reason, difference in availability between terrestrial and undersea links is limited. 


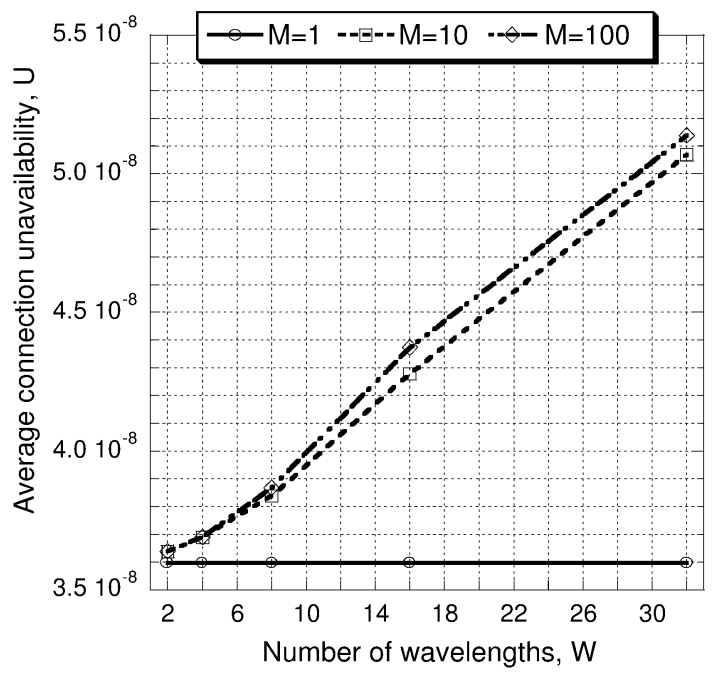

Fig. 6. Average connection unavailability of ITNet; DPP case (results of ACPRO are labeled with $M=x$ ).

The set of connection requests has been built with the help of Telecom Italia Laboratory according to realistic traffic measurements. It comprises 250 unidirectional asymmetric optical connection requests. Two sets of design and optimization experiments have been carried out, for the two path-protection techniques considered in the paper: DPP and SPP. In each of the two sets, the following set of values of $W$ has been scanned: $\{2,4,8,16,32\}$.

The following output performance parameters have been measured:

- average connection unavailability $U$;

- total number of fibers F;

- total number of WDM channels $C$, used by either working or protection lightpaths.

It is worth noting at this point that $C$ does not correspond merely to the product $F \cdot W$, because $C$ represents only the number of used channels and does not count the free residual channel capacity.

For each protection case, the network has been initially dimensioned by performing the MCAD. Then, the ACPRO has been run three times (separately), adopting the following values for the margin $M: M=1$ (unchanged unavailability compared with MCAD), $M=10$ and $M=100$.

Fig. 6 refers to DPP. It displays the average connection unavailability $U$ plotted as a function of $W$.

Resource utilization, the other objective function of the design process, is dealt by Fig. 7(a) and (b), which plots the two output parameters $C$ and $F$ as functions of $W$.

In the three graphs, results of MCAD have not been represented, since they are coincident with those of ACPRO with $M=1$. Being the network capacity unconstrained, MCAD is able to actually find the highest available RFWA for each connection (when possible, all the connections avoid submarine links, which have a higher unavailability). Once MCAD has completed its job, ACPRO with $M=1$ can do very little to reduce the number of fibers. It can reroute connections only if it is able to find alternative cycles with the same unavailability,

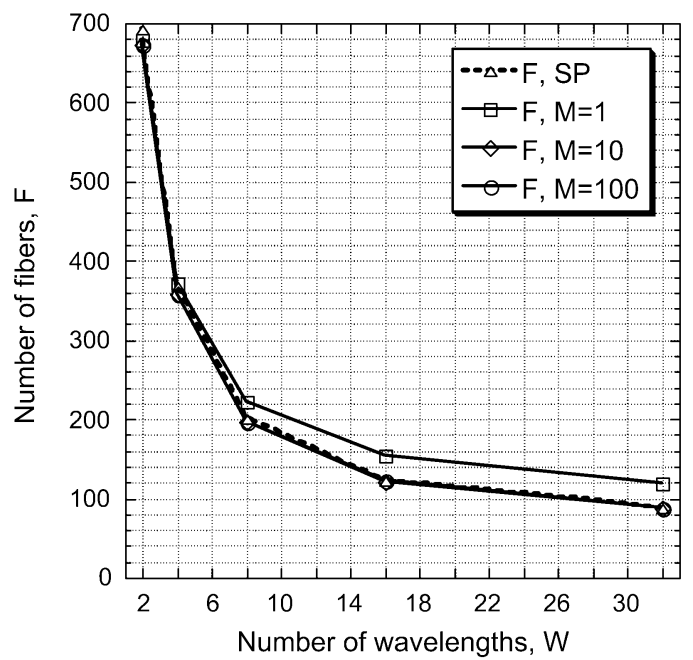

(a)

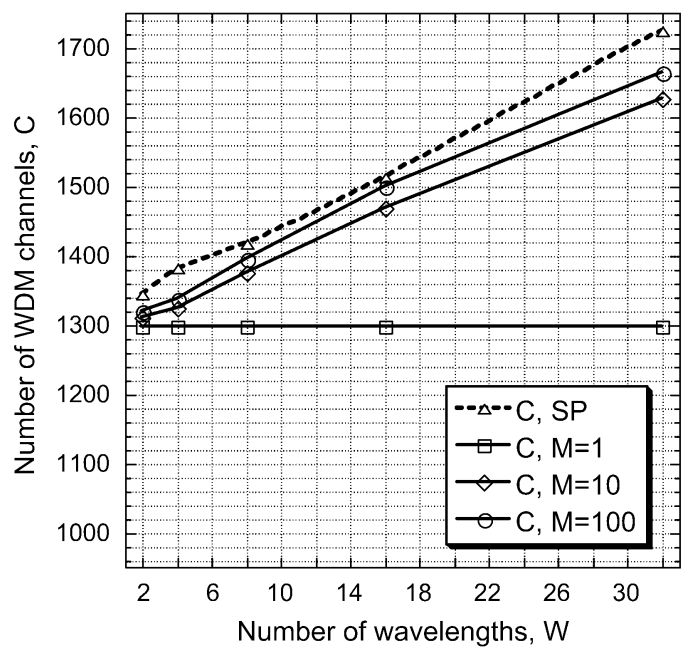

(b)

Fig. 7. (a) Fiber deployment and (b) channel occupation of ITNet; DPP case (SP refers to optimization with no availability constraint and shortest-path routing).

which is practically impossible even for highly connected networks as ITNet. In the DPP case, thus, and for any values of $W, \mathrm{ACPRO}$ results in no gain in the number of deployed WDM channels, no change in the number of occupied WDM channels and obviously no worsening in availability.

It should be noted that the minimum average unavailability obtained by MCAD is fully independent of $W$ (Fig. 6). This again is a consequence of a capacity-unbounded problem and of the fact that availability weights, in our model, are completely independent on the number of wavelengths per fiber. The development of a more accurate model keeping WDM channel density into account in evaluating the availability of transmission systems, will be the subject of future work.

When the margin $M$ is relaxed to 10 and 100, ACPRO is able to reduce the number of deployed fibers, as it appears in Fig. 7(a), where the results for SP and for ACPRO with $M$ equal to 10 and 100 are almost overlapped. The number of occupied channels $C$ is shown in Fig. 7(b), and it increases when the constraint on availability is relaxed. The reason of this increasing 

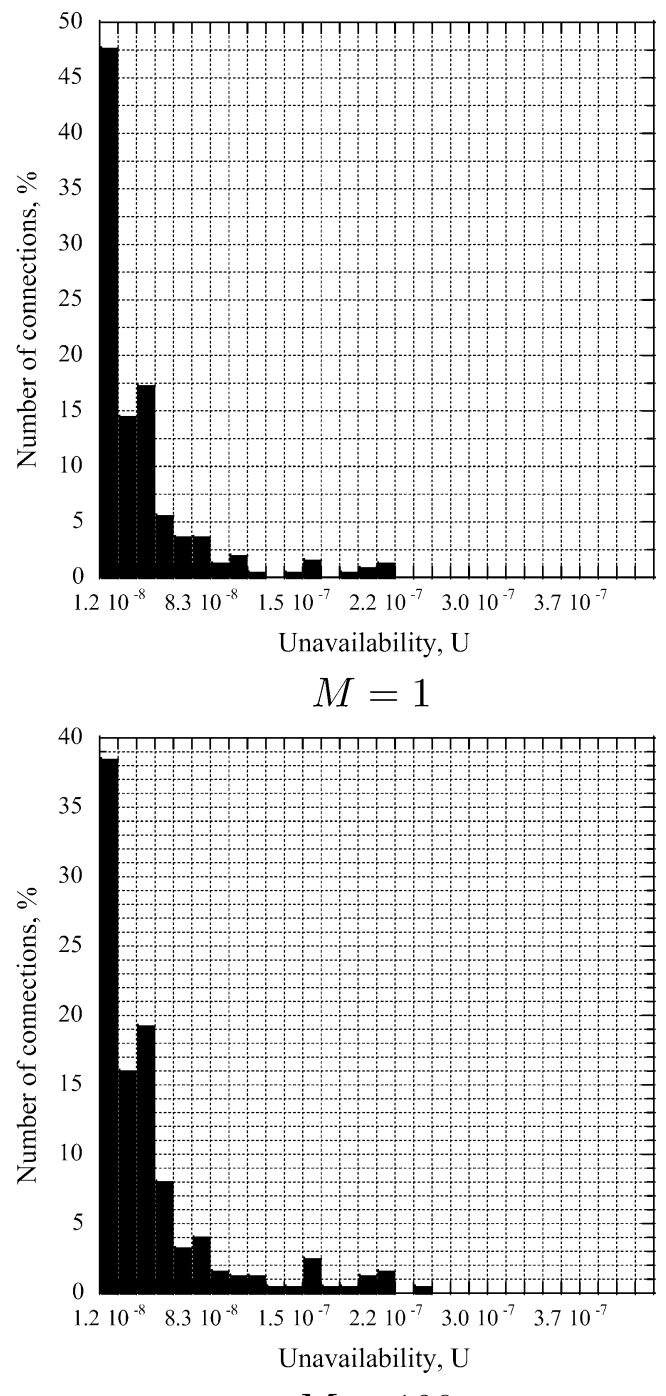

$M=100$

Fig. 8. Connection unavailability histograms of ITNet resulting from ACPRO with $W=16$, DPP case.

trend is that the ACPRO algorithm essentially reroutes connections on longer lightpaths in order to free and remove fibers. This is allowed by the relaxation of the bound on the unavailability for all the connections. There is, however, just a small increase of the average unavailability value (less than a factor 2, far less than the margin), indicating that only few connections undergo rerouting. Rerouting on longer cycles, besides being effective in freeing some partially used fibers, has also the opposite consequence of increasing the total number of consumed channels: resource optimization results from a tradeoff between these two trends.

By comparison, we have reported on the graph also the results of optimization performed without considering any constraint on availability and adopting a new routing criterion, instead of an availability metric: the objective is to minimize the number of fibers recurring to shortest-path-based algorithm [21], and we refer to this strategy as SP. With margins 10 and 100, ACPRO converges almost perfectly to the SP solution. The increase in the number of deployed WDM channels is an effect of WDM granularity. If we suppose that all the connections are routed

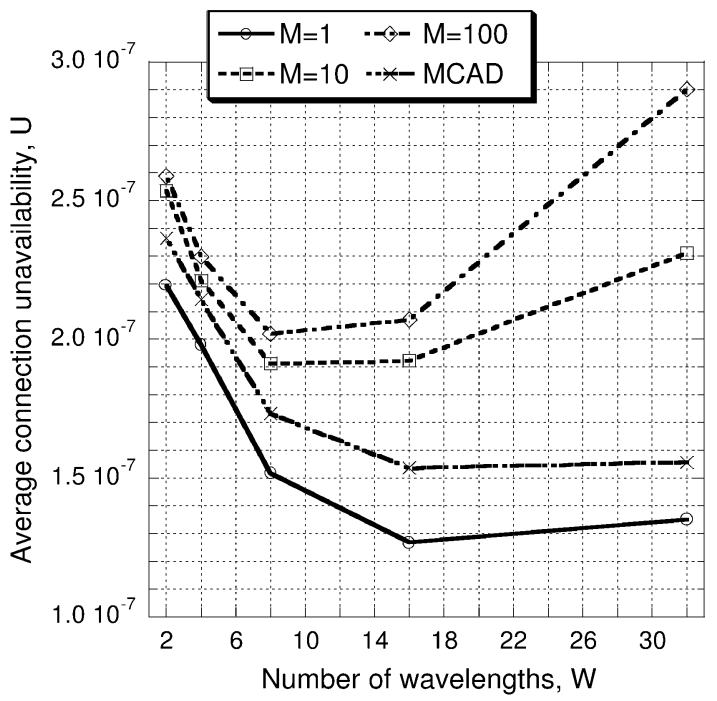

Fig. 9. Average connection unavailability of ITNet; SPP case (MCAD refers to minimum unavailability routing).

along their shortest cycle, the corresponding $\bar{C}$ is the smallest possible channel occupation (which for ITNet loaded with our static traffic is around 1340 channels). The lower bound on the number of deployed channels is $\bar{D}=W \cdot\lceil\bar{C} / W\rceil$, which is an increasing function of $W$. In conclusion, with DPP in the ITNet case even a small margin is sufficient to optimize network resources; a larger margin is useless.

Fig. 8 displays the probability density histograms of the availability values of the connections for the ITNet with DPP after ACPRO has been performed with margin 1 and 100, with $W=$ 16. As a further proof of what was stated above, we notice how after ACPRO few connections appear with higher unavailability, while the shape of the probability density function does not change very much.

Let us now switch to SPP. Results obtained designing ITNet are displayed in Figs. 9 and 10.

The curves are quite different from the dedicated case. Resource-sharing implies a general unavailability increase of about one order of magnitude. On the other hand, the number of occupied channels decreases of about 300 (roughly from 20\% to $30 \%$ ), while the number of installed fibers decreases by $20 \%$ for $W=2$ and by a small percentage for $W=32$.

The MCAD curve $U$ is no longer flat, but it has a minimum around $W=16$. Availability decreases compared with DPP due to sharing. As the MCAD SPP $C$ curve [Fig. 10(b)] confirms, for the value $W=16$ at which unavailability is minimum, channel occupation has a maximum, indicating a low sharing degree.

The ACPRO curve with $M=1$ does not coincide with MCAD any more. Surprisingly, at a first sight, ACPRO with $M=1$ reaches lower unavailability values then MCAD. The explanation is that in the attempt of minimizing resources, ACPRO, by rerouting, forces many connections to return from sharing to dedicated path protection. This is proved also by the fact that ACPRO with $M=1$ occupies more channels than MCAD and is successful in reducing the number of fibers [Fig. 10(a)].

The difference in terms of unavailability between the results of ACPRO with $M=10$ and $M=100$ is more relevant than in 


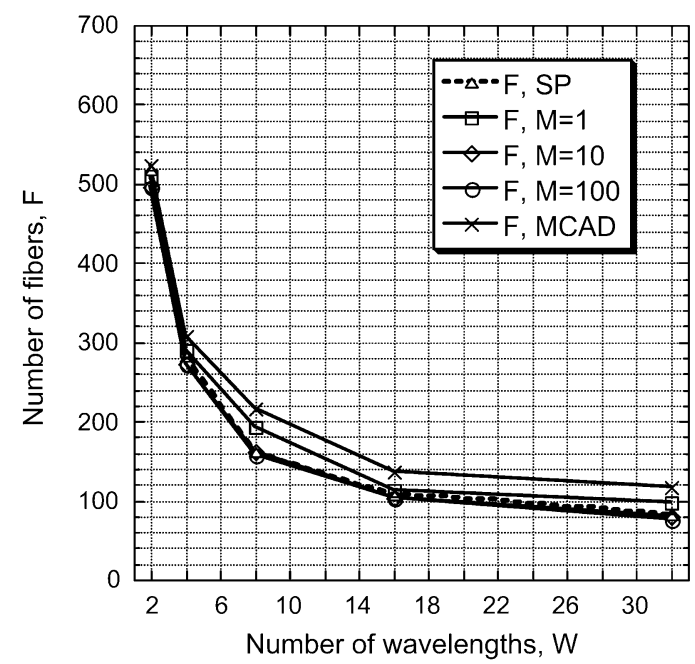

(a)

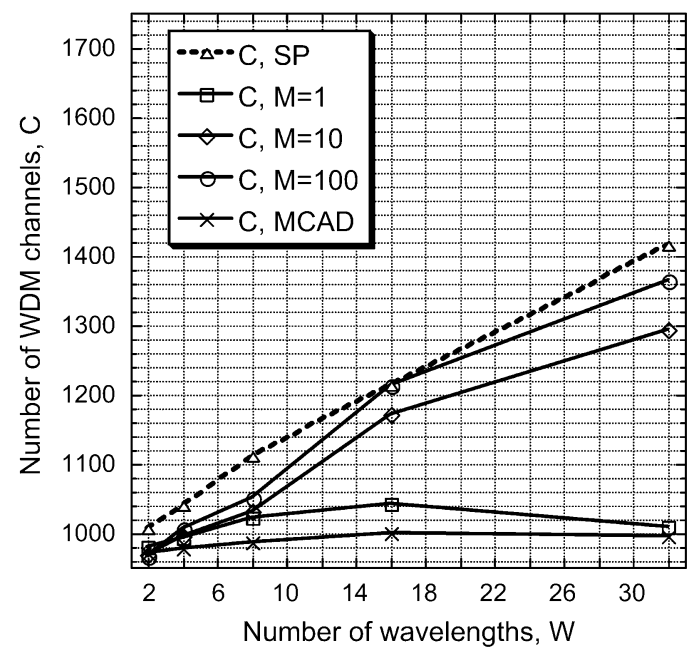

(b)

Fig. 10. (a) Fiber and (b) channels deployment and occupation of ITNet; SPP case.

the dedicated case. The increments compared with MCAD and ACPRO with $M=1$ indicate an increased use of sharing. As (4) shows, unavailability of some connections rapidly increases as their sharing groups get populated. The chance of sharing are increased by the relaxation of the availability constraint that allows to route connections along longer cycles. The average unavailability remains, however, well below the threshold set by the margin even in this SPP case. In fact, the values of $F$ and $C$ obtained by ACPRO with margins 10 and 100 are again very close to the corresponding values of the shortest-path optimization. This indicates that even with SPP, relaxing availability constraints beyond a certain level does not help in further reducing physical resources.

Fig. 11 displays the probability density histograms of the availability values of the connections for the ITNet with DPP after ACPRO has been performed with margin 1 and 100, with $W=16$.

The second case-study network we have considered is the well known U.S. National Science Foundation Network (NSFNet), represented in Fig. 12.

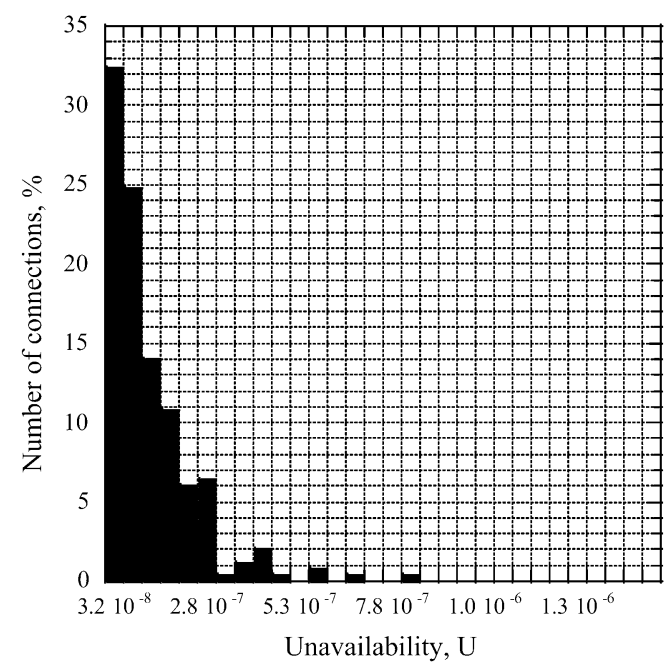

$M=1$

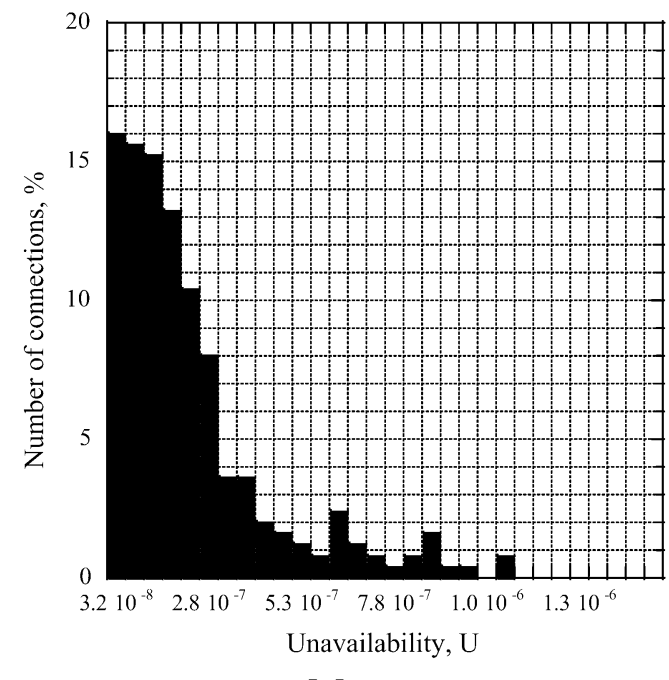

$M=100$

Fig. 11. Connection unavailability histograms of ITNet resulting from ACPRO with $W=16$, SPP case.

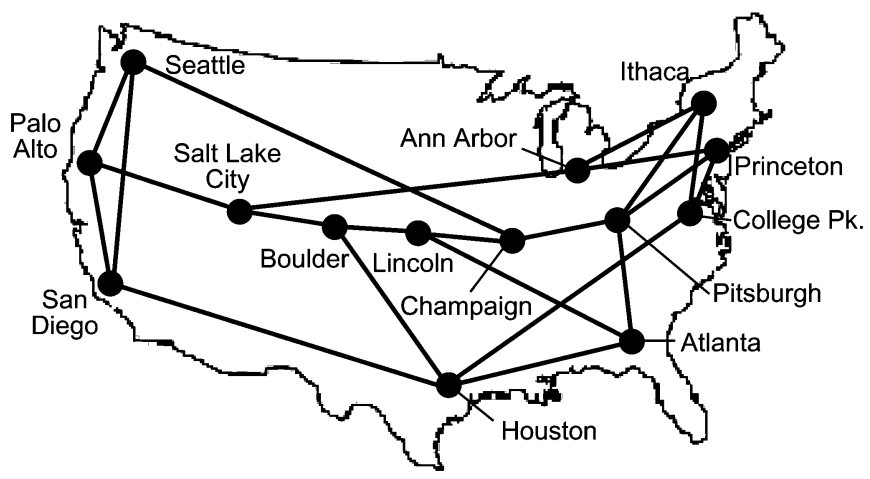

Fig. 12. National Science Foundation Network (NSFNet).

The WDM channel availability model is the same adopted for the ITNet, described in Section IV. NSFNet has however only terrestrial links, a much larger geographical extension and lower connectivity, with 14 nodes and 22 links. Traffic demand is composed of 360 unidirectional connection requests, distributed in 


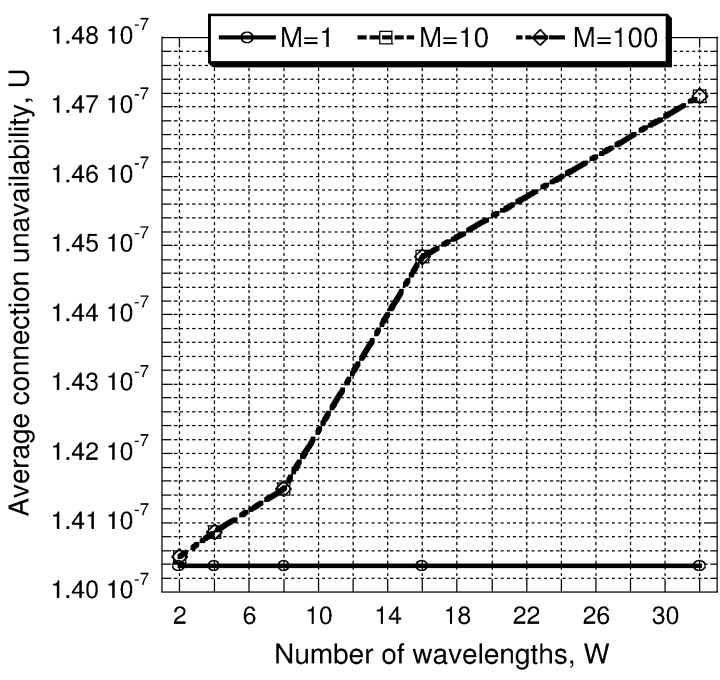

(a)

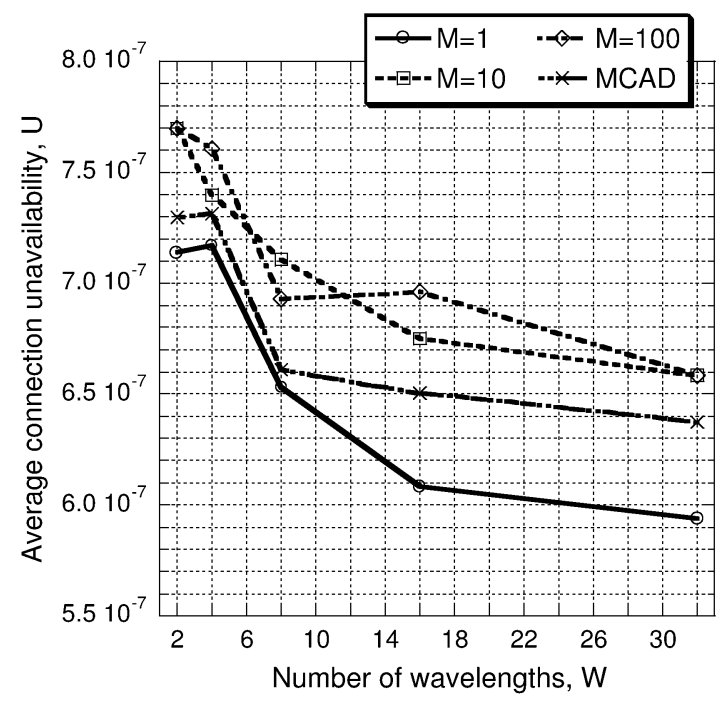

(c)

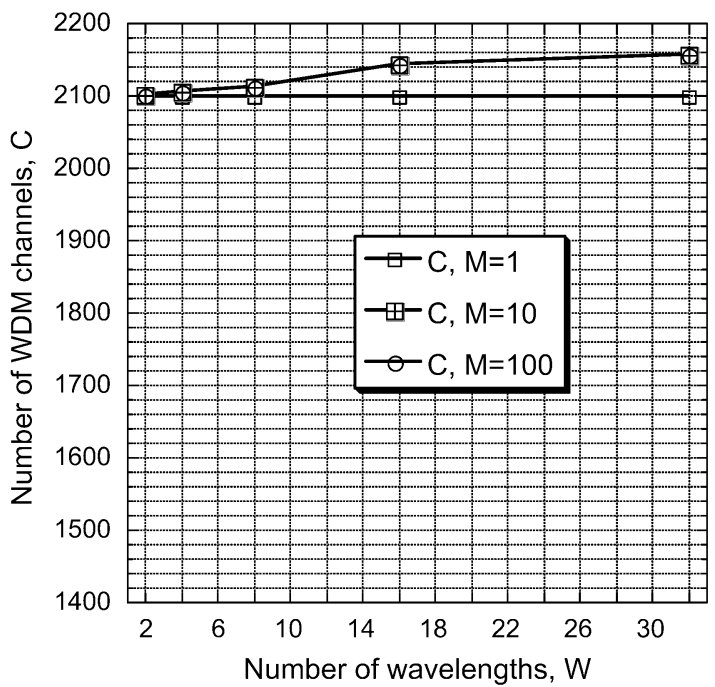

(b)

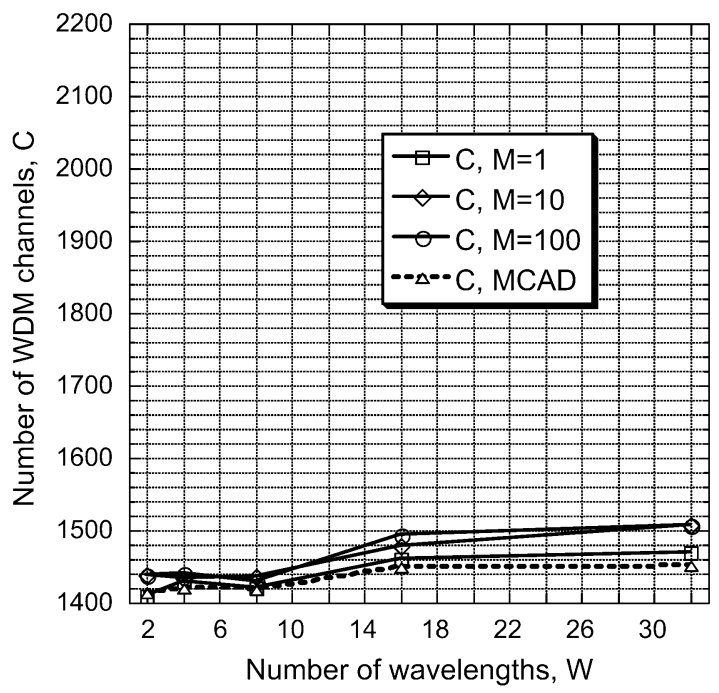

(d)

Fig. 13. NSFNet design: average connection unavailability in (a) DPP case and (c) SPP case; channel deployment and occupation in (b) DPP case and (d) SPP case.

order that traffic is symmetric, derived from realistic traffic measurements reported in [29].

Fig. 13 displays all the results concerning NSFNet dimensioning and optimization.

The curves plotted in the graphs have behaviors very similar to those of the ITNet, confirming with another example all the observations we have previously stated. The major difference with the ITNet is a global worsening of the unavailability performance, due to the larger geographical extension of this second network.

From Fig. 13(a), we can further observe that ACPRO with $M=10$ and $M=100$ gives exactly the same results and that the difference between the highest and lowest unavailability is very small (about $7 \cdot 10^{-9}$, while it was $1.5 \cdot 10^{-8}$ for ITNet). NSFNet traffic is dominated by few source-destination node pairs demanding a very high number of connections: this makes the network very "tight" after MCAD (many unused fibers are removed), with few chances of rerouting.
In Fig. 13(c) a crossover takes place between the two curves $U$ of ACPRO with margins 10 and 100 for $W=8$. For that particular value of $W$, ACPRO with $M=100$ is able to find a planning solution that occupies a small number of WDM channels [though hardly visible in Fig. 13(d)] and, thus, has a high average availability. The corresponding total number of fibers (or of deployed channels $D$ ) follows, however, the general trend given by ACPRO for other values of $W$ (see curve $C$ with $M=100$ ). It should also be noted that curves in Fig. 13(c), unlike the corresponding curves for ITNet, do not have a minimum around $W=16$ : the sharing degree gradually decreases with $W$, instead of having a peak, as confirmed by channel occupation [Fig. 13(d)].

\section{A. Traffic Expansion Under Fixed Link Capacities}

So far, we have considered static traffic matrices, exploring a viable method to ensure guaranteed availability levels, while minimizing the network resources. Due to new 


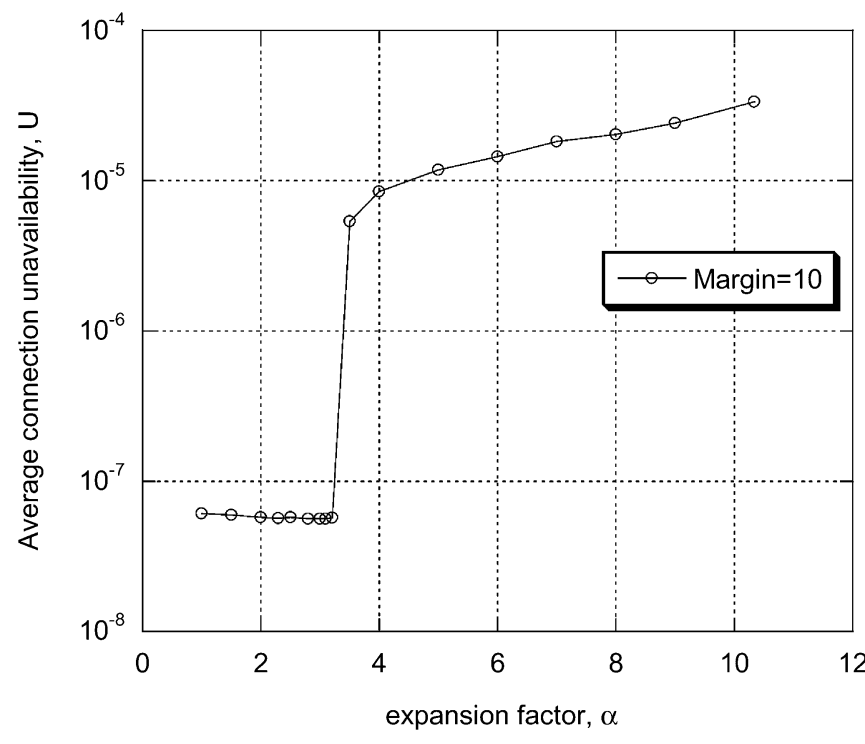

Fig. 14. Average connection unavailability for increasing values of scaling traffic factor $\alpha$.

bandwidth-hungry services, transport networks have been experimenting uninterrupted traffic growth: in Fig. 14, we show on ITNet how the average unavailability is affected by a traffic volume increase, keeping the physical resource allocation fixed after the initial design phase of the network. The whole traffic matrix is multiplied by a factor $\alpha$ in order to increase its dimension. To apply a fair scaling to the traffic matrix, we have defined a new basic traffic matrix, which corresponds to $\alpha=1$ : this new matrix respects the original size relations among the various entries, but each entry has a value greater or equal to one. Then, the matrix has been multiplied by increasing values of the scaling factor $\alpha$, assigning to each entry the value of the closest integer. We have fixed a fiber assignment on ITNet as follows: each terrestrial (submarine) link is equipped with 24 (13) fibers, each fiber supports eight wavelength channel. We have then applied ACPRO with a margin equal to 10 to allocate connection requests. Fig. 14 shows that the average unavailability value is constant until $\alpha=3.2$, when the value of $U$ suddenly increase of about two order of magnitude. The reason of this behavior is that $\alpha=3.2$ is the highest value of $\alpha$ for which the network resource can accommodate all the traffic, according to ACPRO. Over that value, connections that cannot be protected are routed on unprotected working lightpath or rejected if no admissible working path is found. Due to the new unprotected connections in the network the average availability value suddenly worsen.

In conclusion, a correct design of the network is a critical factor to guarantee the required availability level of the optical connections. ACPRO has been shown to successfully achieve this requirement, jointly addressing capacity and availability objectives.

\section{CONCLUSION AND FUTURE WORK}

In this paper, we have presented a heuristic method to design and optimize an optical transport network according to a given set of static protected connections, so that two objectives are pursued: obtaining high availability of the connections and minimizing the network deployment cost. Algorithms and techniques have been provided to support availability metric as a routing criterion. Optimization procedures have been proposed to optimize the network when dedicated path protection and shared path protection are adopted as protection techniques in the OTN layer. Case-study analysis has been carried out by solving some examples of optimization, starting from realistic availability data, network topologies, and traffic. Results show that the typical "five-nines" target (unavailability order of magnitude of $10^{-5}$ ) can easily be achieved in a first phase of lightpath allocation in a capacity-unconstrained network model. We have considered only internal failure causes related to realistic equipment availability. External failures, such as fiber cuts, which are not controllable by the technology provider and which strongly depend on deployment characteristics, have not been taken into account, although their inclusion in the model is possible.

Good network resource minimization can result from a subsequent heuristic optimization phase, without severely compromising connection availability. By exploiting this procedure the network can be designed with a total amount of fibers not far from the theoretical lower bound for the given traffic. Sharing of preplanned protection resources proved to be a good technique to reduce the network cost, while achieving an acceptable reliability performance.

Another aspect of the problem is the role of availability in the dynamic decision-making and failure management for rerouting and failure management. Availability information could be used by a standard link-state routing protocol to efficiently provision reliable connection. So, further study is needed to develop availability-guaranteed provisioning framework to support on-the fly decisions.

\section{ACKNOWLEDGMENT}

The authors would like to thank A. Righetti (CoreCom), M. Villa (Pirelli Optical Systems), and R. Clemente (Telecom Italia Laboratory) for their precious collaboration in case-study and availability parameter definition. Great appreciation goes also to D. Arci and D. Petecchi for the active collaboration in the software developments.

\section{REFERENCES}

[1] A. Fumagalli, M. Tacca, F. Unghvary, and A. Farago, "Shared path protection with differentiated reliability," in Proc. IEEE Int. Conf. Commun., vol. 4, Apr. 2002, pp. 2157-2161.

[2] E. E. Lewis, Introduction to Reliability Engineering, J. W. Sons, Ed. New York: Wiley, 1987.

[3] A. Kiss, G. Vesztergombi, J. Levendovszky, and L. Jereb, "Adaptative statistical algorithms in network reliability analysis," in Proc. Int. Conf. Telecommun. Syst., 2001, pp. 426-435.

[4] L. Jereb, T. Jakab, and F. Unghvary, "Availability analysis of multi-layer optical networks," Opt. Netw. Mag., pp. 84-94, Mar--Apr. 2002.

[5] M. Clouqueur and W. D. Grover, "Availability analysis of span-restorable mesh networks," IEEE J. Sel. Areas Commun., vol. 20, no. 4, pp. 810-821, May 2002.

[6] A. Antonoupolos, J. J. O. Reilly, and P. Lane, "A framework for the availability assessment of SDH transport networks," in Proc. 2nd IEEE Symp. Comput. Commun., Jul. 1997, pp. 666-670.

[7] D. A. Schupke, A. Autenrieth, and T. Fisher, "Survivability of multiple fiber duct failures," in Proc. 3rd Int. Workshop Design Reliable Commun. Netw. (DRCN), Oct. 7-10, 2001, pp. 219-231. 
[8] M. Son and B.-C. Jeon, "Reliable optical networks-Fault management aspects," in Proc. Int. Workshop Design Reliable Commun. Netw. (DRCN), Oct. 7-10, 2001, pp. 9-14.

[9] M. Tornatore, G. Maier, A. Pattavina, M. Villa, A. Righetti, R. Clemente, and M. Martinelli, "Availability optimization of static path-protected WDM networks," in Proc. Opt. Fiber Commun. Conf. (OFC), Mar. 2003, pp. 621-622.

[10] R. Ramamurthy, J.-F. Labourdette, A. Akyamach, and S. Chaudhury, "Limiting sharing on protection channels in mesh optical networks," in Proc. Opt. Fiber Commun. Conf. (OFC), vol. 1, Mar. 17-22, 2003, pp. 204-205.

[11] J. Doucette, M. Clouqueur, and W. D. Grover, "On the availability and capacity requirements of shared backup path-protected mesh networks," Opt. Netw. Mag., vol. 4, no. 6, pp. 29-44, 2003.

[12] J. Zhang, K. Zhu, and B. Mukherjee, "Service provisioning to provide per-connection-based availability guarantee in WDM mesh networks," in Proc. Opt. Fiber Commun. Conf. (OFC), vol. 2, Mar. 2003, pp. 622-624.

[13] Y. Huang, W. Wen, J. Heritage, and B. Mukherjee, "A generalized protection framework using a new link state availability model for reliable optical networks," IEEE/OSA J. Lightw. Technol., vol. 22, pp. 2536-2547, Nov. 2004.

[14] L. Song, J. Zhang, and B. Mukherjee, "Dynamic provisioning with reliability guarantee and resource optimization for differentiated services in WDM mesh networks," in Proc. Opt. Fiber Commun. Conf. (OFC), Mar. 2005, p. OWG4.

[15] A. M. Mood, D. C. Boes, and F. A. Graybill, Introduction to the Theory of Statistics, 3rd ed. New York: McGraw-Hill, 1974.

[16] C. Y. Lee, "Analysis of switching networks," Bell Syst. Tech. J., vol. 34, pp. 1287-1315, Nov. 1955.

[17] H. Waldman, D. Campelo, and R. Almeida, "A new analytical approach for the estimation of blocking probabilities in wavelength routing networks," in Proc. OPTICOMM, 2002, pp. 324-335.

[18] D. Arci, D. Petecchi, G. Maier, A. Pattavina, and M. Tornatore, "Availability models for protection techniques in WDM networks," in Proc. Int. Workshop Design Reliable Commun. Netw. (DRCN), 2003, pp. $158-166$.

[19] R. Clemente, L. Serra, G. D'Orazio, and G. Cosmo, "A framework for class of service definition in GMPLS-based meshed ASTN," in Proc. Int. Workshop Design Reliable Commun. Netw. (DRCN), 2003, pp. 93-100.

[20] G. Maier, A. Pattavina, L. Roberti, and T. Chich, "A heuristic approach for the design of static multifiber WDM networks: Principles and applications," Opt. Netw. Mag., vol. 3, no. 5, pp. 52-66, Sep./Oct. 2002.

[21] A. Dacomo, S. D. Patre, G. Maier, A. Pattavina, and M. Martinelli, "Design of static resilient WDM mesh networks with multiple heuristic criteria," in Proc. IEEE INFOCOM, Jun. 2002, pp. 1793-1802.

[22] R. Bhandari, Survivable Networks Algorithms for Diverse Routing. Norwell, MA: K. A. Publishers, 1999.

[23] M. Tornatore, G. Maier, and A. Pattavina, "Maximum availability routing with dedicated path protection," Politecnico di Milano and CoreCom, Milano, Paper in preparation.

[24] R. Ramamurthy, Z. Bogdanowicz, S. Samieian, D. Saha, B. Rajagopalan, S. Sengupta, S. Chaudhuri, and K. Bala, "Capacity performance of dynamic provisioning in optical networks," J. Lightw. Technol., vol. 19, pp. 40-48, Jan. 2001.

[25] H. Zang, C. Ou, and B. Mukherjee, "Path-protection routing and wavelength assignment (RWA) in WDM mesh networks under duct-layer constraints," IEEE/ACM Trans. Netw., vol. 11, pp. 248-258, Apr. 2003.

[26] P. Sebos, J. Yates, G. Hjalmtysson, and A. Greenberg, "Auto-discovery of shared risk link groups," in Proc. Opt. Fiber Commun. Conf. (OFC), vol. 3, 2001, pp. WDD3-1-WDD3-3.
[27] S. Ou, J. Zhang, H. Zhang, L. Sahasrabuddhe, and B. Mukherjee, "New and improved approaches for shared path protection in WDM mesh networks," IEEE/OSA J. Lightw. Technol., vol. 22, no. 5, pp. 1223-1332, May 2004.

[28] K. C.-H. Chu, M. Mezhoudi, and Y. Hu, "Comprehensive end-to-end reliability assessment of optical network transports," in Proc. Opt. Fiber Commun. Conf. (OFC), Mar. 17-22, 2002, pp. 228-230.

[29] Y. Miyao and H. Saito, "Optimal design and evolution of survivable WDM transport networks," IEEE J. Sel. Areas Commun., vol. 16, pp. 1190-1198, Sep. 1999.

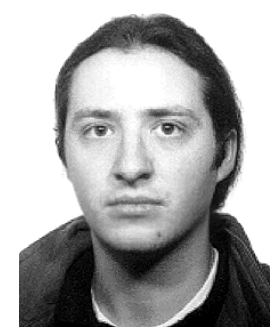

Massimo Tornatore (S'02) received the Laurea degree in telecommunications engineering from Politecnico di Milano, Milan, Italy, in 2001. He is currently working towards the Ph.D. degree in the Electronics and Information Department, Politecnico di Milano under the supervision of Prof. Pattavina.

His research interests include design, protection strategies, traffic grooming in optical WDM networks, and group communication security.

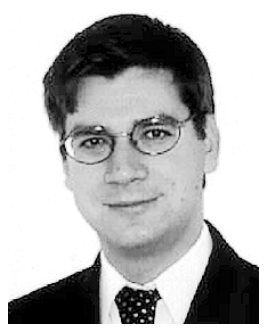

Guido Maier (S'97-M'99) received the Laurea degree in electronic engineering and the Ph.D. degree in telecommunication engineering from Politecnico di Milano, Milan, Italy, in 1995 and 2000, respectively.

$\mathrm{He}$ is a Researcher at CoreCom, Milan, Italy, where he holds the position of Head of the Optical Networking Laboratory. He is currently involved in industrial and European research projects. He is an author of more than 30 papers in the area of optical networks published in international journals and conference proceedings. His main areas of interest are optical network modeling, design and optimization, ASON/GMPLS architecture, and WDM switching systems.

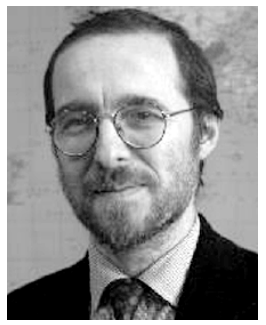

Achille Pattavina (S'85-SM'93) received the Dr.Eng. degree in electronic engineering from the University "La Sapienza," Rome, Italy, in 1977.

He was with the University "La Sapienza" until 1991 when he moved to the Politecnico di Milano, Milan, Italy, where he is now a Full Professor. $\mathrm{He}$ is author of more than 100 papers in the area of communications networks published in international journals and conference proceedings. He is author of the book Switching Theory, Architectures and Performance in Broadband ATM Networks (New York: Wiley, 1998). His current research interests are in the area of optical networks and wireless networks.

Dr. Pattavina is a Senior Member of the IEEE Communications Society. He has been an Editor for Switching Architecture Performance of the IEEE TRANSACTIONS ON COMMUNICATIONS since 1994 and Editor-in-Chief of the European Transactions on Telecommunications since 2001. 